\title{
AMOSTRAGEM E VARIABILIDADE ESPACIAL DE CARACTERÍSTICAS QUÍMICAS DE UM LATOSSOLO SUBMETIDO A DIFERENTES SISTEMAS DE PREPARO ${ }^{1}$
}

\author{
PEDRO MARQUES DA SILVEIRA ${ }^{2}$, FRANCISCO JOSÉ PFEILSTICKER ZIMMERMANN ${ }^{3}$, \\ SILVANDO CARLOS DA SILVA ${ }^{4}$ e ADRIANY ALVES DA CUNHA ${ }^{5}$
}

\begin{abstract}
RESUMO - O trabalho foi conduzido na Embrapa-Centro Nacional de Pesquisa de Arroz e Feijão, em Santo Antônio de Goiás, GO, em Latossolo Vermelho-Escuro distrófico, textura argilosa, submetido a diferentes sistemas de preparo, durante cinco anos consecutivos (1992-1996), e cultivado com milho no verão e feijoeiro no inverno, sob irrigação por aspersão. O objetivo foi avaliar as características químicas de um solo Latossolo Vermelho-Escuro após cinco anos de uso de três sistemas de preparo para o plantio. Os sistemas foram: com arado de aiveca, grade aradora e plantio direto. As amostras para análise química foram coletadas, em todos os três tratamentos, em uma malha quadrada de 49 pontos (7x7), a espaços de $4 \mathrm{~m}$ x $4 \mathrm{~m}$, e nas profundidades de $0-5 \mathrm{~cm}$ e $5-20 \mathrm{~cm}$ de solo. As amostras foram analisadas para determinação do $\mathrm{pH}, \mathrm{Ca}, \mathrm{Mg}, \mathrm{P}, \mathrm{K}$ e cálculo da saturação por bases. Em relação a cada variável calculou-se o valor médio, mínimo, máximo e coeficiente de variação, comparando-se as médias, entre tratamentos, pelo teste t. Os valores de $\mathrm{pH}, \mathrm{Ca}, \mathrm{Mg}, \mathrm{P}, \mathrm{K}$ e saturação por bases do solo variaram nos diferentes tratamentos. Na profundidade de $0-5 \mathrm{~cm}$, os valores de todas as variáveis foram maiores no sistema plantio direto do que no arado e na grade. Os valores de $\mathrm{P}$ e de $\mathrm{K}$ apresentaram as maiores variabilidades, e os de $\mathrm{pH}$, as menores.
\end{abstract}

Termos para indexação: características físico-químicas do solo, nutrientes minerais, $\mathrm{pH}$ do solo, arado de aiveca, grade aradora, plantio direto.

\section{SAMPLING SIZE AND SPATIAL VARIABILITY OF CHEMICAL PROPERTIES OF A LATOSOL SUBJECTED TO DIFFERENT SOIL PREPARATIONS}

\begin{abstract}
The study was conducted at the Embrapa-Centro Nacional de Pesquisa de Arroz e Feijão, in Santo Antônio de Goiás, GO, Brazil, on a clayey Oxisol subjected to different soil tillage systems for five consecutive years and cultivated with corn in the summer and bean in the winter under sprinkler irrigation. The objective of this study was to determine the effects of three soil tillage systems, using moldboard plough, harrow disc and no-tillage. Forty nine soil samples were collected from a $7 \times 7$ lattice sampling area spaced $4 \mathrm{~m} \mathrm{x} 4 \mathrm{~m}$ at 0-5 cm and 5-20 cm soil depth. Soil samples were analyzed for $\mathrm{pH}, \mathrm{Ca}, \mathrm{Mg}, \mathrm{P}, \mathrm{K}$ and base saturation. Minimum, maximum and average values were calculated along with the coefficient of variation and average values were compared by the $t$ test. Values of $\mathrm{pH}, \mathrm{Ca}, \mathrm{Mg}, \mathrm{P}, \mathrm{K}$ and base saturation of soil varied for different treatments. All soil chemical properties values were higher at $0-5 \mathrm{~cm}$ soil depth in no-tillage treatment as compared to harrow disc and moldboard plough treatments. Among the soil chemical properties evaluated, P and K concentrations showed the highest variability, whereas $\mathrm{pH}$ presented the lowest.
\end{abstract}

Index terms: soil chemicophysical properties, mineral nutrients, soil $\mathrm{pH}$, moldboard plough, disc harrows, direct sowing.

\footnotetext{
${ }^{1}$ Aceito para publicação em 3 de janeiro de 2000.

2 Eng. Agrôn., Dr., Embrapa-Centro Nacional de Pesquisa de Arroz e Feijão (CNPAF), Caixa Postal 179, CEP 75375-000 Santo Antônio de Goiás, GO. Bolsista do CNPq. E-mail: pmarques@cnpaf.embrapa.br

${ }^{3}$ Eng. Agrôn., Ph.D., Embrapa-CNPAF.

E-mail: fjpz@cnpaf.embrapa.br

${ }^{4}$ Eng. Agrôn., M.Sc., Embrapa-CNPAF.

E-mail: silvando@cnpaf.embrapa.br

${ }^{5}$ Eng. Agrôn., Embrapa-CNPAF. Bolsista do CNPq.
}

\section{INTRODUÇÃO}

Existe bastante variabilidade nas características químicas e físicas de um solo, mesmo em uma área uniforme segundo suas características visíveis de campo, como: topografia, cor e vegetação. Para que a amostragem do solo para fins agrícolas represente com exatidão a sua fertilidade, é necessário o conhecimento dessa variabilidade, pois só assim as reco- 
mendações de calagem e adubação não estariam comprometidas. Quanto mais heterogêneo for o solo, maior deve ser o número de amostras coletadas, para que se atinja maior exatidão na avaliação de suas características.

Segundo Santos \& Vasconcellos (1987), o número de amostras para análise química que represente bem uma determinada área pode variar com o manejo do solo, cultura anterior e fertilização da área. Souza (1992) relatou que inúmeros trabalhos têm mostrado que os sistemas de manejo conservacionistas criam um ambiente no solo diferente do encontrado no sistema convencional, resultante dos efeitos dos resíduos superficiais e da reduzida movimentação do solo; como resultado, tem sido encontrado um acúmulo superficial de fertilizantes nos sistemas conservacionistas.

O número mínimo de amostras de solo para estimar o valor médio da variável de interesse com uma determinada exatidão tem sido calculado em vários trabalhos. Segundo Souza (1992), o procedimento consiste em coletar ao acaso um certo número de amostras individuais, analisar, calcular o coeficiente de variação dos dados, achar o valor de tabela do teste $\mathrm{t}$ correspondente ao número de graus de liberdade do quadrado médio residual, estabelecer a diferença permitida em torno da média e, assim, calcular o número mínimo de amostras individuais a serem coletadas em futuras amostragens.

Barreto et al. (1974) verificaram, em um Podzólico Vermelho-Amarelo câmbico, que o número de amostras simples para formar uma amostra composta para determinação dos valores de $\mathrm{pH}, \mathrm{Ca}+\mathrm{Mg}$ e $\mathrm{Al}$ deveriam ser, respectivamente, 1, 19 e 13. Quanto a P e K, há necessidade de, respectivamente, 38 e 19 amostras simples. Vê-se, portanto, que o número de amostras varia em relação aos diferentes elementos químicos do solo.

No que se refere à variação da amostragem de diferentes manejos do solo, Santos \& Vasconcellos (1987), trabalhando em um Latossolo Vermelho-Escuro distrófico, fase cerrado, recomendaram coletar: nove amostras compostas, formadas de dez simples, para áreas recém-desmatadas; sete amostras compostas, formadas de dez simples, para uma área arada e gradeada; e 15 amostras compostas, formadas de dez simples, para uma área que recebeu adubação e calagem. Esta recomendação considera uma variação máxima de $20 \%$ em torno da média e $80 \%$ de probabilidade, aplicável aos elementos que apresentaram maior variabilidade no solo.

Este trabalho teve como objetivo avaliar as características químicas de um Latossolo Vermelho-Escuro, após cinco anos de emprego de três sistemas de preparo para plantio.

\section{MATERIAL E MÉTODOS}

O trabalho foi conduzido em um Latossolo VermelhoEscuro distrófico, textura argilosa, na Fazenda Capivara, da Embrapa-Centro Nacional de Pesquisa de Arroz e Feijão, em Santo Antônio de Goiás, GO. A área foi cultivada durante cinco anos consecutivos (1992-1996) com as culturas de milho no verão e feijoeiro no inverno, sob irrigação por aspersão, sistema pivô central. Essas culturas foram semeadas em solo sob três tratamentos de preparo: (1) arado de aiveca; (2) grade aradora e (3) plantio direto.

No primeiro tratamento, usou-se um arado de três aivecas, de 30,5 cm de comprimento, incorporando-se ao solo os resíduos das culturas até a profundidade de $30 \mathrm{~cm}$, seguindo-se uma gradagem com grade destorroadora.

No segundo tratamento, usou-se uma grade aradora de 20 discos, de $66 \mathrm{~cm}$ de diâmetro, incorporando ao solo os resíduos até $15 \mathrm{~cm}$.

No terceiro tratamento, o plantio direto foi feito com semeadora apropriada, permanecendo na superfície do solo todo o resíduo das culturas. A adubação empregada no plantio de cada cultura foi de $400 \mathrm{~kg} / \mathrm{ha}$, da fórmula 5-30-5.

No quarto ano de plantio, foram aplicadas $2,5 \mathrm{t} / \mathrm{ha}$ de calcário, em toda a área experimental. Nos tratamentos arado e grade, o corretivo foi incorporado ao solo; e no plantio direto, permaneceu na superfície. A precipitação pluvial ocorrida nos anos de 1992, 1993, 1994, 1995 e 1996 foi de, respectivamente, $1.521,1.116,1.676,1.487$ e $1.145 \mathrm{~mm}$, concentrados principalmente nos meses de outubro a março.

As amostras para análise química foram coletadas, em todos os tratamentos, em 49 pontos de uma malha quadrada de $7 \times 7$ pontos, com espaços, ente si, de $4 \mathrm{~m} \mathrm{x} 4 \mathrm{~m}$, e nas profundidades de $0-5 \mathrm{~cm}$ e $5-20 \mathrm{~cm}$. A coleta em profundidade teve como objetivo a avaliação do acúmulo superficial de fertilizantes. As amostras foram analisadas com vistas à determinação do $\mathrm{pH}, \mathrm{P}, \mathrm{K}, \mathrm{Ca}, \mathrm{Mg}$, e cálculo da saturação por bases (V) do solo, conforme metodologia da Embrapa (1997). Os dados foram analisados calculandose o valor médio, mínimo, máximo e coeficiente de variação (C.V.). Os valores médios das variáveis foram comparados pelo teste $\mathrm{t}$. 
Determinaram-se também os números de subamostras necessárias para compor uma amostra composta e estimar o valor médio das variáveis, usando-se a fórmula: $\mathrm{N}=(\mathrm{T} \alpha \times \mathrm{C} . \mathrm{V} .)^{2} / \mathrm{D}$.....(equação 1$)$

em que: $\mathrm{N}=$ número mínimo de amostras; $\mathrm{T} \alpha=$ valor do teste $\mathrm{t}$ de Student para o nível de probabilidade de $95 \%$; C.V. = coeficiente de variação; $\mathrm{e} \mathrm{D}=$ porcentagem de variação em torno da média $(5,10,15,20,25$ e $30 \%)$.

Foi feita, também, a espacialização dos dados de campo, do $\mathrm{pH}$ e do teor de $\mathrm{P}$ do solo, pelo sistema de informações geográficas desenvolvido pelo Instituto Nacional de Pesquisas Espaciais (1990).

\section{RESULTADOS E DISCUSSÃO}

Os valores médios, mínimos, máximos e coeficientes de variação dos dados de $\mathrm{pH}, \mathrm{Ca}, \mathrm{Mg}, \mathrm{P}, \mathrm{Ke} \mathrm{V}$ em função dos tratamentos, são apresentados nas Tabelas 1 e 2, respectivamente, para as profundidades de 0-5 cm (primeira camada) e 5-20 cm (segunda camada) de solo. Quanto ao $\mathrm{pH}$, houve diferença significativa entre os tratamentos, nas duas profundidades estudadas. Na profundidade de $0-5 \mathrm{~cm}$, o maior valor de $\mathrm{pH}$ ocorreu no plantio direto, o qual foi estatisticamente diferente dos encontrados nos tratamentos com arado e grade. Já na camada de
5-20 cm, o sistema plantio direto apresentou o menor valor de $\mathrm{pH}$, também diferente dos demais tratamentos. Na primeira camada, as amplitudes observadas em relação ao $\mathrm{pH}$ foram de 1,0,1,3 e 1,5, respectivamente, para o arado, grade e plantio direto. Os coeficientes de variação foram baixos com relação a esta variável, em todos os tratamentos, variando de $3,7 \%$ a $5,4 \%$, o que evidencia a baixa variabilidade dessa característica. Prevedello (1987) também encontrou baixo C.V. em relação ao $\mathrm{pH}$, em torno de $3 \%$, quando estudou a magnitude da variabilidade espacial de um solo Terra Roxa Estruturada cultivado com arroz de sequeiro.

A maior amplitude observada no plantio direto explica muito bem o maior coeficiente de variação neste tratamento em relação aos demais. Em razão do não-revolvimento do solo, como ocorre no plantio direto, espera-se maior variabilidade dos dados neste tratamento. Segundo Souza (1992), o sistema convencional usando grade aradora, em virtude do revolvimento do solo, apresenta menor variabilidade, horizontal e vertical, nos teores de matéria orgânica e nutrientes na camada arada do solo do que a escarificação e plantio direto, entre os quais a escarificação é mais variável, por movimentar apenas algumas faixas no solo.

TABELA 1. Valores médios, mínimos, máximos, coeficiente de variação e número mínimo de subamostras necessário para estimar o $\mathrm{pH}, \mathrm{Ca}, \mathrm{Mg}, \mathrm{P}, \mathrm{K}$ e V em várias porcentagens de variação em torno da média, nos tratamentos arado, grade e plantio direto, na profundidade de $0-5 \mathrm{~cm}$ de $5010^{1}$.

\begin{tabular}{|c|c|c|c|c|c|c|c|c|c|c|c|}
\hline \multirow[t]{2}{*}{ Variável } & \multirow[t]{2}{*}{ Tratamento } & \multirow[t]{2}{*}{ Média } & \multirow[t]{2}{*}{ Mínimo } & \multirow[t]{2}{*}{ Máximo } & \multirow[t]{2}{*}{ C.V. } & \multicolumn{6}{|c|}{$\begin{array}{c}\text { Número de subamostras referente à variação } \\
\text { em torno da média }\end{array}$} \\
\hline & & & & & & $5 \%$ & $10 \%$ & $15 \%$ & $20 \%$ & $25 \%$ & $30 \%$ \\
\hline \multirow[t]{3}{*}{$\mathrm{pH}\left(\mathrm{H}_{2} \mathrm{O}\right)$} & Arado & $5,9 b$ & 5,3 & 6,3 & 4,0 & 3 & 1 & 1 & 1 & 1 & 1 \\
\hline & Grade & $5,8 b$ & 5,4 & 6,7 & 4,1 & 3 & 1 & 1 & 1 & 1 & 1 \\
\hline & P. direto & $6,4 a$ & 5,4 & 6,9 & 5,4 & 5 & 2 & 1 & 1 & 1 & 1 \\
\hline \multirow[t]{3}{*}{$\mathrm{Ca}^{2+}\left(\mathrm{cmol}_{\mathrm{c}} \mathrm{dm}^{-3}\right)$} & Arado & $2,7 b$ & 1,7 & 4,0 & 19,6 & 67 & 17 & 8 & 5 & 3 & 2 \\
\hline & Grade & $2,7 b$ & 1,8 & 3,7 & 16,8 & 48 & 12 & 6 & 3 & 2 & 2 \\
\hline & P. direto & $4,5 \mathrm{a}$ & 2,6 & 5,9 & 18,2 & 58 & 15 & 7 & 4 & 3 & 2 \\
\hline \multirow[t]{3}{*}{$\mathrm{Mg}^{2+}\left(\mathrm{cmol}_{\mathrm{c}} \mathrm{dm}^{-3}\right)$} & Arado & $0,9 b$ & 0,4 & 1,6 & 25,6 & 106 & 27 & 12 & 7 & 5 & 3 \\
\hline & Grade & $0,9 b$ & 0,4 & 2,0 & 28,8 & 134 & 34 & 15 & 9 & 6 & 4 \\
\hline & P. direto & $1,3 \mathrm{a}$ & 0,6 & 1,9 & 21,6 & 75 & 19 & 9 & 5 & 3 & 3 \\
\hline \multirow[t]{3}{*}{$\mathrm{P}\left(\mathrm{mg} \mathrm{dm}^{-3}\right)$} & Arado & $9,1 \mathrm{c}$ & 1,0 & 25,8 & 59,8 & 572 & 143 & 64 & 36 & 23 & 16 \\
\hline & Grade & $21,9 b$ & 10,3 & 34,8 & 22,1 & 79 & 20 & 9 & 5 & 4 & 3 \\
\hline & P. direto & $39,8 \mathrm{a}$ & 5,8 & 112,8 & 63,2 & 640 & 160 & 72 & 40 & 26 & 18 \\
\hline \multirow[t]{3}{*}{$\mathrm{K}^{+}\left(\mathrm{mg} \mathrm{dm}^{-3}\right)$} & Arado & $79 \mathrm{c}$ & 42 & 194 & 36,3 & 211 & 53 & 24 & 14 & 9 & 6 \\
\hline & Grade & $105 b$ & 25 & 189 & 40,6 & 264 & 66 & 30 & 17 & 11 & 8 \\
\hline & P. direto & $147 \mathrm{a}$ & 65 & 292 & 28,5 & 131 & 33 & 15 & 9 & 6 & 4 \\
\hline \multirow[t]{3}{*}{$\mathrm{V}(\%)$} & Arado & $38 b$ & 27 & 52 & 16,8 & 46 & 12 & 6 & 3 & 2 & 2 \\
\hline & Grade & $35 c$ & 24 & 48 & 15,0 & 37 & 10 & 5 & 3 & 2 & 1 \\
\hline & P. direto & $55 \mathrm{a}$ & 34 & 74 & 17,6 & 50 & 13 & 6 & 4 & 2 & 2 \\
\hline
\end{tabular}

1 Valores seguidos pela mesma letra não diferem pelo teste $t$, a $5 \%$ de probabilidade. 
TABELA 2. Valores médios, mínimos, máximos, coeficiente de variação e número mínimo de subamostras necessário para estimar o $\mathrm{pH}, \mathrm{Ca}, \mathrm{Mg}, \mathrm{P}, \mathrm{K}$ e V em várias porcentagens de variação em torno da média, nos tratamentos arado, grade e plantio direto, na profundidade de $5-20 \mathrm{~cm}$ de solo ${ }^{1}$.

\begin{tabular}{|c|c|c|c|c|c|c|c|c|c|c|c|}
\hline \multirow[t]{2}{*}{ Variável } & \multirow[t]{2}{*}{ Tratamento } & \multirow[t]{2}{*}{ Média } & \multirow[t]{2}{*}{ Mínimo } & \multirow[t]{2}{*}{ Máximo } & \multirow[t]{2}{*}{ C.V. } & \multicolumn{6}{|c|}{$\begin{array}{l}\text { Número de subamostras referente à variação } \\
\text { em torno da média }\end{array}$} \\
\hline & & & & & & $5 \%$ & $10 \%$ & $15 \%$ & $20 \%$ & $25 \%$ & $30 \%$ \\
\hline \multirow[t]{3}{*}{$\mathrm{pH}\left(\mathrm{H}_{2} \mathrm{O}\right)$} & Arado & $5,9 a$ & 5,4 & 6,3 & 3,7 & 3 & 1 & 1 & 1 & 1 & 1 \\
\hline & Grade & $5,6 b$ & 4,9 & 6,0 & 4,4 & 4 & 1 & 1 & 1 & 1 & 1 \\
\hline & P. direto & $5,4 \mathrm{c}$ & 4,7 & 5,9 & 5,4 & 5 & 2 & 1 & 1 & 1 & 1 \\
\hline \multirow[t]{3}{*}{$\mathrm{Ca}^{2+}\left(\mathrm{cmol}_{\mathrm{c}} \mathrm{dm}^{-3}\right)$} & Arado & $2,6 a$ & 1,7 & 3,6 & 19,8 & 66 & 17 & 8 & 5 & 3 & 2 \\
\hline & Grade & $2,4 b$ & 1,4 & 3,4 & 20,3 & 73 & 19 & 9 & 5 & 3 & 3 \\
\hline & P. direto & $2,1 \mathrm{c}$ & 0,8 & 4,0 & 28,5 & 134 & 34 & 15 & 9 & 6 & 4 \\
\hline \multirow[t]{3}{*}{$\mathrm{Mg}^{2+}\left(\mathrm{cmol}_{\mathrm{c}} \mathrm{dm}^{-3}\right)$} & Arado & $0,9 \mathrm{a}$ & 0,4 & 1,4 & 26,4 & 112 & 28 & 13 & 7 & 5 & 4 \\
\hline & Grade & $0,8 b$ & 0,3 & 1,3 & 29,3 & 138 & 35 & 16 & 9 & 6 & 4 \\
\hline & P. direto & $0,6 \mathrm{c}$ & 0,1 & 1,4 & 37,5 & 225 & 57 & 25 & 15 & 9 & 7 \\
\hline \multirow[t]{3}{*}{$\mathrm{P}\left(\mathrm{mg} \mathrm{dm}^{-3}\right)$} & Arado & $9,3 \mathrm{~b}$ & 0,5 & 36,1 & 70,1 & 787 & 197 & 88 & 50 & 32 & 22 \\
\hline & Grade & $15,0 \mathrm{a}$ & 4,9 & 75,2 & 74,7 & 894 & 224 & 100 & 56 & 36 & 25 \\
\hline & P. direto & $19,6 \mathrm{a}$ & 2,4 & 131,5 & 136,9 & 2998 & 750 & 334 & 188 & 120 & 84 \\
\hline \multirow[t]{3}{*}{$\mathrm{K}^{+}\left(\mathrm{mg} \mathrm{dm}^{-3}\right)$} & Arado & $86 a$ & 47 & 223 & 38,4 & 236 & 59 & 27 & 15 & 10 & 7 \\
\hline & Grade & $98 \mathrm{a}$ & 20 & 172 & 33,3 & 178 & 45 & 20 & 12 & 8 & 5 \\
\hline & P. direto & $53 b$ & 25 & 137 & 50,7 & 412 & 103 & 46 & 26 & 17 & 12 \\
\hline \multirow[t]{3}{*}{$\mathrm{V}(\%)$} & Arado & $38 \mathrm{a}$ & 28 & 52 & 16,7 & 45 & 12 & 5 & 3 & 2 & 2 \\
\hline & Grade & $33 b$ & 21 & 44 & 16,2 & 42 & 11 & 5 & 3 & 2 & 2 \\
\hline & P. direto & $27 \mathrm{c}$ & 11 & 43 & 25,5 & 104 & 26 & 12 & 7 & 5 & 3 \\
\hline
\end{tabular}

${ }^{1}$ Valores seguidos pela mesma letra não diferem pelo teste $\mathrm{t}$, a $5 \%$ de probabilidade.

Com relação ao $\mathrm{Ca}$ e ao $\mathrm{Mg}$, os maiores valores ocorreram no plantio direto na primeira camada, e os menores, na segunda, também no plantio direto. A maior concentração de Ca e de $\mathrm{Mg}$ no plantio direto, nos primeiros $5 \mathrm{~cm}$ da camada arável, mostrando uma real concentração desses elementos na camada superficial do solo, é decorrência da calagem feita no quarto ano de cultivo, sem incorporação do corretivo neste tratamento.

A análise estatística mostrou diferença nos teores de $\mathrm{P}$ do solo entre os tratamentos, nas duas camadas de solo. Em ambas, os maiores valores de $\mathrm{P}$ ocorreram no plantio direto, e os menores, no tratamento com arado. O tratamento com grade apresentou valores intermediários. Os menores valores no tratamento com arado são decorrentes do revolvimento provocado pela ação do implemento, causando a diluição do nutriente pela mistura com maior volume de solo, o que melhor explica a redução do teor de P nas duas camadas. Já no plantio direto houve concentração dos teores do elemento na primeira camada. Acumulações superficiais de $\mathrm{P}$ no plantio direto, sobretudo nos primeiros $5 \mathrm{~cm}$ de profundidade de dois solos, Latossolo Roxo distrófico e Latossolo Vermelho-Escuro distrófico argiloso, foram encontradas por Muzilli (1983). Segundo o autor, esse acúmulo se explica pela imobilidade e baixa solubilidade dos compostos de P, sobretudo em solos de natureza ácida e com altos teores de argila e metais pesados. Na camada de 10-20 cm, os valores de P solúvel encontrados por Muzilli (1983) em plantio convencional foram superiores aos do plantio direto, sugerindo, assim, um efeito de incorporação do fertilizante em profundidade pela inversão da camada arável por meio das operações de preparo.

Segundo Vasconcellos et al. (1982), a amostragem de solo em áreas com adubação no sulco de plantio, efetuada após o preparo mecânico do terreno com aração e gradagem, pode minimizar o erro amostral ocasionado pela concentração do fertilizante fosfatado. Essa afirmativa é endossada pelos resultados obtidos neste trabalho.

Os valores mínimo e máximo de $\mathrm{P}$ retratam bem os altos coeficientes de variação obtidos em relação ao nutriente. Na primeira camada de solo, os tratamentos arado e grade apresentaram menores amplitudes de variação, respectivamente iguais a $24,8 \mathrm{mg} \mathrm{dm}^{-3} \mathrm{e}$ 
$24,5 \mathrm{mg} \mathrm{dm}^{-3}$. A maior amplitude de variação, $107 \mathrm{mg} \mathrm{dm}^{-3}$, ocorreu no plantio direto e, por conseguinte, o maior coeficiente de variação $(63,2 \%)$. Neste tratamento e nesta camada, o valor máximo de $\mathrm{P}$ $\left(112,8 \mathrm{mg} \mathrm{dm}^{-3}\right)$ foi 19,4 vezes o valor mínimo $\left(5,8 \mathrm{mg} \mathrm{dm}^{-3}\right)$. Na segunda camada, o plantio direto apresentou ainda maior amplitude de variação, igual a $129,1 \mathrm{mg} \mathrm{dm}^{-3} \mathrm{e}$, por conseguinte, maior C.V. $(136,9 \%)$. Os altos valores de $\mathrm{P}$, de $112,8 \mathrm{mg} \mathrm{dm}^{-3} \mathrm{em}$ 0-5 $\mathrm{cm}$ e de $131,5 \mathrm{mg} \mathrm{dm}^{-3}$ em 5-20 cm, devem ser oriundos da coincidência da coleta de solo nas linhas de plantio e de adubação das culturas instaladas anteriormente na área, fato muito comum na coleta de amostras de solo sob plantio direto.

Quanto ao K, houve efeito significativo dos tratamentos nos teores médios do elemento nas duas profundidades de solo. Na profundidade de $0-5 \mathrm{~cm}$, o maior valor ocorreu no plantio direto, seguido, em ordem, pela grade e pelo arado. Novamente, os menores valores no arado são decorrentes da inversão da camada superficial do solo, feita pelo tratamento, como explicado anteriormente quando se discutiu o P. O sistema plantio direto apresentou o maior valor de K na primeira camada, mas o menor, na segunda, o que mostra que o cátion concentrou-se na camada superficial do solo. Igual concentração ocorreu também quanto aos cátions $\mathrm{Ca}$ e $\mathrm{Mg}$, como visto anteriormente, e, provavelmente, devido à fixação desses dois elementos pelas cargas negativas da superfície do solo. Resultados semelhantes, com relação à K, $\mathrm{Ca}$ e $\mathrm{Mg}$ e a um maior valor de $\mathrm{pH}$, na camada de 0-5 cm, em relação à camada de 5-20 cm de profundidade, foram encontrados por Muzilli (1983).

Foram encontrados altos valores de $\mathrm{K}$, em todos os tratamentos e profundidades, atingindo $292 \mathrm{mg} \mathrm{dm}^{-3}$ no plantio direto. Neste tratamento, a amplitude de variação alcançou $227 \mathrm{mg} \mathrm{dm}^{-3}$ na profundidade de 0-5 $\mathrm{cm}$.

Houve diferença significativa dos tratamentos no valor médio de $\mathrm{V}$, o qual foi maior no plantio direto na primeira camada, e menor, na segunda. Os valores encontrados na primeira camada foram, de modo geral, superiores aos da segunda. O maior valor da saturação por bases, igual a 74\%, ocorreu no plantio direto. Os valores do C.V. desta variável foram menores que os observados com relação a P e K, mostrando, assim, que esta propriedade do solo tem menor variabilidade do que a desses dois elementos.

Pela equação 1 , calculou-se o número mínimo de amostras, para estimar os valores de $\mathrm{pH}, \mathrm{Ca}, \mathrm{Mg}, \mathrm{P}$, $\mathrm{K}$ e V dos diferentes tratamentos, com relação a diferentes porcentagens de variação em torno da média $(5,10,15,20,25$ e $30 \%)$, também apresentados nas Tabelas 1 e 2 , respectivamente, no que se refere às profundidades de $0-5 \mathrm{~cm}$ (primeira camada) e $5-20 \mathrm{~cm}$ (segunda camada) de solo. Considerando que o número mínimo de subamostras de solo é diretamente proporcional ao coeficiente de variação, quanto maior o C.V., maior será o número de subamostras a serem coletadas. Quanto ao $\mathrm{pH}$, pelo baixo C.V. dos dados, a variável pode ser estimada com número relativamente pequeno de subamostras. Para uma variação de $5 \%$ em torno da média, o número mínimo de subamostras para os tratamentos arado, grade e plantio direto foi de, respectivamente, 3, 4 e 5, números bastante exequíiveis na prática. Com relação a Ca e $\mathrm{Mg}$, considerando a mesma variação (5\%), os números foram maiores. Quanto ao $\mathrm{P}$, os números variaram de 79 no tratamento grade $(0-5 \mathrm{~cm})$ a 2.998 no plantio direto $(5-20 \mathrm{~cm})$. No que tange ao $\mathrm{K}$, o menor valor encontrado foi igual a 131 no plantio direto $(0-5 \mathrm{~cm})$. Esses valores são praticamente inexeqüíveis, tanto em relação ao $\mathrm{P}$ quanto em relação ao K.

Barreto et al. (1974) encontraram o número de subamostras igual a 3, para estimar o valor de $\mathrm{pH}$, numa variação de $5 \%$ em torno de um resultado médio representativo de um Podzólico Vermelho-Amarelo câmbico fase terraço. Já em relação a P e K, os números encontrados foram 601 e 2.226. Números elevados de subamostras referentes a $\mathrm{P}$ e $\mathrm{K}$, na mesma porcentagem de segurança, também foram encontrados por Souza (1992). Forsythe (1970), estudando a importância da variabilidade das propriedades do solo, citou a necessidade de 760 subamostras para estimar o valor de $\mathrm{K}$ do solo, ainda assim com $10 \%$ de variação em torno da média.

Utilizando-se o procedimento de se coletar 20 subamostras, como proposto pela Comissão de Fertilidade do Solo do Estado de Minas Gerais (1989) para o Estado de Minas Gerais e pela Comissão de Fertilidade do Solo - RS/SC (1995) para os Estados de Santa Catarina e Rio Grande do Sul, os resultados obtidos mostram que os teores de $\mathrm{Ca}(5-20 \mathrm{~cm})$ esta- 
riam sendo estimados em cerca de $10 \%$ de variação em torno da média, nos tratamentos arado e grade, e de $15 \%$ no plantio direto. Quanto ao $\mathrm{Mg}$, a estimativa com 20 subamostras estaria em torno de $15 \%$ nos tratamentos arado e grade e de $10 \%(0-5 \mathrm{~cm})$ a $20 \%$ $(5-20 \mathrm{~cm})$ no plantio direto.

Já para o P, na camada de $0-5 \mathrm{~cm}$, a estimativa estaria variando de $10 \%$ em torno da média no tratamento grade e de $30 \%$ no arado e no plantio direto. De acordo com os dados médios de P, $30 \%$ em torno da média significa que os valores estariam variando entre $6,8 \mathrm{mg} \mathrm{dm}^{-3}$ e $11,4 \mathrm{mg} \mathrm{dm}^{-3}$ no tratamento com arado e de $27,9 \mathrm{mg} \mathrm{dm}^{-3}$ a $51,7 \mathrm{mg} \mathrm{dm}^{-3}$ no plantio direto. Na camada de 5-20 cm de profundidade, com 20 subamostras, a variação em torno da média seria superior a $30 \%$ em todos os tratamentos.

Com esta mesma quantidade de subamostras, a estimativa de variação em torno da média de K estaria variando de $15 \%$ (tratamento grade) a $25 \%$ (plantio direto), ambos na camada de $5-20 \mathrm{~cm}$.

Para o caso da saturação por bases, em função dos menores coeficientes de variação dessa propriedade do solo, amostras compostas formadas de 20 amostras simples implicariam uma porcentagem de variação em torno do valor médio menor que $10 \%$ referente a todos os tratamentos, com exceção do plantio direto, a 5-20 $\mathrm{cm}$ de profundidade.

De acordo com Souza (1992), a importância do erro de amostragem na estimativa do valor de uma característica do solo, em face do número de subamostras utilizado, está em razão do valor esperado para aquela característica. Assim, quando se espera obter um teor de P no solo bem acima do nível crítico daquele solo, pode-se admitir um maior desvio percentual da média, resultante de um baixo número de subamostras, já que este fato pouco influirá na decisão da adubação. Mas, se os valores esperados estão abaixo do nível crítico, uma estimativa mais precisa deve ser buscada, procurando coletar um maior número de subamostras, pois erros de estimativa poderão deslocar o valor obtido para outra faixa de recomendação, e assim, provocar adubações maiores ou menores que a necessária.

A distribuição espacial dos valores originais do $\mathrm{pH}$ do solo, em todos os sistemas de preparo e nas duas profundidades $(0-5 \mathrm{~cm}$ e $5-20 \mathrm{~cm})$, está apresentada na Fig. 1. Os valores foram agrupados nas faixas de $\mathrm{pH}$ de até 5,6 (vermelho), 5,7 a 5,8 (amarelo), 5,9 a 6,0 (azul) e maior que 6,1 (verde). Observa-se, no sistema plantio direto, valores maiores de $\mathrm{pH}$ do solo (cor verde) na camada de $0-5 \mathrm{~cm}$ em relação a 5-20 cm, caracterizando, assim, maior variabilidade dos valores da variável em profundidade, dentro das faixas preestabelecidas. Entretanto, o tratamento apresentou menor variabilidade horizontal, identificada pela maior uniformidade das cores na Fig. 1. No tratamento com arado, pode-se observar menor variabilidade dos valores de $\mathrm{pH}$ entre as duas camadas de solo, provavelmente pela ação
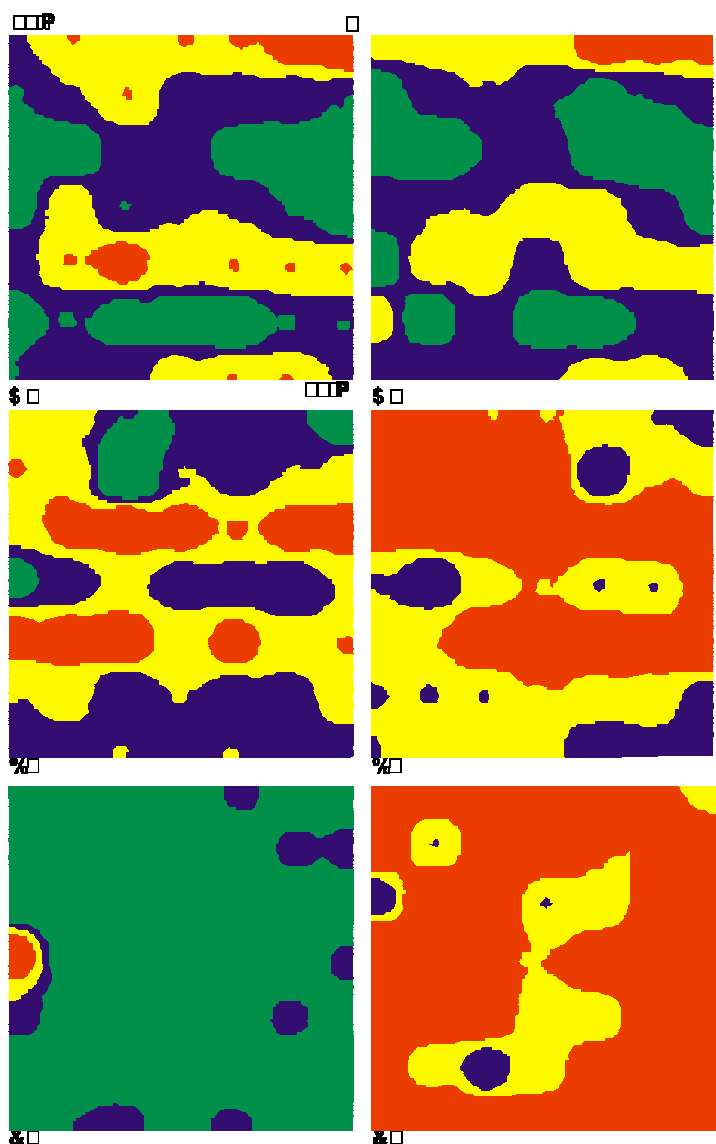

$\square \leqslant \square \square \quad \square \square \square 00$

- $\square 000000$

$\mathbf{0} \geq 00$

FIG. 1. Distribuição espacial dos valores de pH do solo nos diferentes sistemas de preparo e profundidade de amostragem (A: arado; B: grade; C: plantio direto; $1: 0-5 \mathrm{~cm}$; 2: $5-20 \mathrm{~cm}$ de profundidade). 
uniformizadora do implemento, no processo de revolvimento dessas camadas. No caso do $\mathrm{P}$ (Fig. 2), os valores foram agrupados nas faixas de até $10,0 \mathrm{mg} \mathrm{dm}^{-3}$ (vermelho), 10,1 a 15,0 $\mathrm{mg} \mathrm{dm}^{-3}$ (amarelo), 15,1 a 20,0 $\mathrm{mg} \mathrm{dm}^{-3}$ (azul) e maior que $20,1 \mathrm{mg} \mathrm{dm}^{-3}$ (verde). Semelhante ao ocorrido com o $\mathrm{pH}$, observa-se, no sistema plantio direto, valores maiores de $\mathrm{P}$ do solo (cor verde) na camada de 0-5 cm em relação a 5-20 cm, caracterizando maior variabilidade dos valores da variável em profundidade. Já com relação ao tratamento com arado, pode-se notar maior uniformidade dos valores de $\mathrm{P}$ entre as duas camadas de solo.
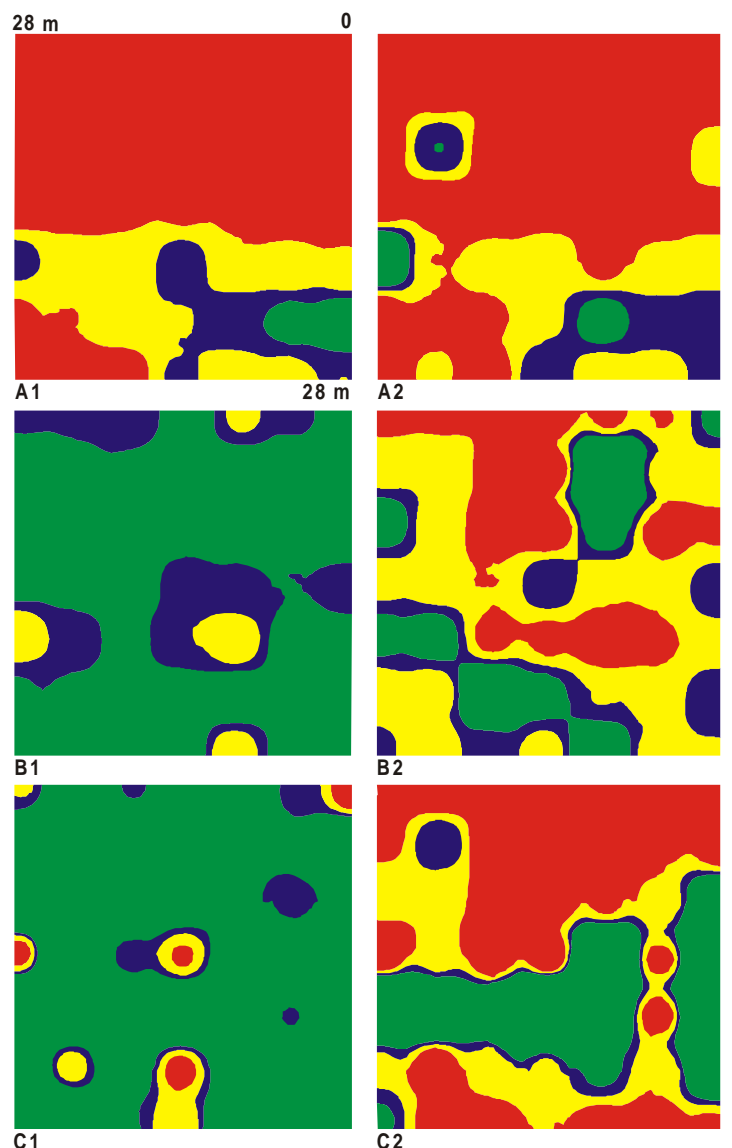

$\square \leqslant 10,0$ $\square 10,1-15,0$

$15,1-20,0$

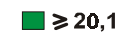

FIG. 2. Distribuição espacial dos valores de $P$ do solo $\left(\mathrm{mg} \mathrm{dm}^{-3}\right)$ nos diferentes sistemas de preparo e profundidade de amostragem (A: arado; $B$ : grade; C: plantio direto; $1: 0-5 \mathrm{~cm}$; 2: $5-20 \mathrm{~cm}$ de profundidade).

\section{CONCLUSÕES}

1. Os valores médios de $\mathrm{pH}, \mathrm{Ca}, \mathrm{Mg}, \mathrm{P}, \mathrm{K}$ e saturação por bases do solo variam conforme o método de preparo e profundidade do solo.

2. Na profundidade de $0-5 \mathrm{~cm}$ de solo, os valores de $\mathrm{pH}, \mathrm{Ca}, \mathrm{Mg}, \mathrm{P}, \mathrm{K}$ e saturação por bases do solo são maiores no sistema plantio direto do que no arado e na grade.

3. Os valores de $\mathrm{P}$ e de $\mathrm{K}$ apresentam as maiores variabilidades, e os de $\mathrm{pH}$, as menores.

4. Para uma variação máxima de $5 \%$ em torno da média, os valores de $\mathrm{pH}$ do solo, nos tratamentos arado, grade e plantio direto, podem ser obtidos utilizando-se baixo número de subamostras; entretanto, para $\mathrm{P}$ e $\mathrm{K}$ com baixo número de subamostras, a variação dos valores em torno da média pode atingir mais de $25 \%$.

\section{REFERÊNCIAS}

BARRETO, A.C.; NOVAIS, R.F.; BRAGA, J.M. Determinação estatística do número de amostras simples de solo para avaliação de sua fertilidade. Revista Ceres, Viçosa, v.21, n.114, p.142-147, 1974.

COMISSÃO DE FERTILIDADE DO SOLO - RS/SC (Passo Fundo, RS). Recomendações de adubação e calagem para os Estados do Rio Grande do Sul e Santa Catarina. 3.ed. Passo Fundo : Sociedade Brasileira de Ciência do Solo/Embrapa-CNPT, 1995. $223 p$.

COMISSÃO DE FERTILIDADE DO SOLO DO ESTADO DE MINAS GERAIS (Lavras, MG). Recomendações para o uso de corretivos e fertilizantes em Minas Gerais: 4ª aproximação. Lavras, 1989. 176p.

EMBRAPA. Centro Nacional de Pesquisa de Solos (Rio de Janeiro, RJ). Manual de métodos de análise de solo. 2.ed.rev.atual. Rio de Janeiro : Embrapa-CNPS, 1997. 212p. (Embrapa-CNPS. Documentos, 1).

FORSYTHE, W.M. Importancia de la variabilidad de las propiedades del suelo para evaluarlas en su manejo. Turrialba, San Jose, v.20, n.4, p.445-451, 1970.

INSTITUTO NACIONAL DE PESQUISAS ESPACIAIS (São José dos Campos, SP). Manual do usuário do SGI. São José dos Campos, 1990. 1v.

Pesq. agropec. bras., Brasília, v.35, n.10, p.2057-2064, out. 2000 
MUZILLI, O. Influência do sistema de plantio direto, comparado ao convencional, sobre a fertilidade da camada arável do solo. Revista Brasileira de Ciência do Solo, Campinas, v.7, n.1, p.95-102, 1983.

PREVEDELLO, B.M.S. Variabilidade espacial de parâmetros de solo e planta. Piracicaba : ESALQ, 1987. 166p. Tese de Doutorado.

SANTOS, H.L.; VASCONCELLOS, C.A. Determinação do número de amostras de solo para análise química em diferentes condições de manejo. Revista Brasi- leira de Ciência do Solo, Campinas, v.11, n.2, p.97$100,1987$.

SOUZA, L.S. Variabilidade espacial do solo em sistemas de manejo. Porto Alegre : UFRGS, 1992. 162p. Tese de Doutorado.

VASCONCELLOS, C.A.; SANTOS, H.L.; BAHIA FILHO, A.F.C.; OLIVEIRA, A.C.; PACHECO, E.B. Amostragem de solo em área com adubação fosfatada aplicada a lanço e no sulco de plantio. Revista Brasileira de Ciência do Solo, Campinas, v.6, n.3, p.221-225, 1982. 